\title{
Combination of post-fascia iliaca compartment block and dexmedetomidine in pain and inflammation control after total hip arthroplasty for elder patients: a randomized control study
}

\author{
Xiaofen Liu, Xianwen Hu, Rui Li and Ye Zhang*
}

\begin{abstract}
Objective: This study aims to investigate the efficiency of fascia iliaca compartment block (FICB) combined with dexmedetomidine (DEX) in postoperative and inflammation management for elder patients after total hip arthroplasty.

Methods: The present randomized controlled study included a total of 119 elder patients who received total hip arthroplasty in our hospital from March 2016 to December 2018. These patients were divided into three groups: control group, patients received routine general anesthesia; FICB group, patients received additional FICB after surgery; and combined group, patients received both pre-treatment of DEX and post-treatment of fascia iliaca compartment block. The serum levels of interleukin (IL)-1 $\beta, I L-6$, and C-reactive protein (CRP) were measured by ELISA. The visual analog scale (VAS) score was measured at $12,24,48$, and $72 \mathrm{~h}$ after surgery, and the patientcontrolled intravenous analgesia (PCIA) pressing time within $48 \mathrm{~h}$ after surgery was also recorded. The Pittsburgh sleep quality index (PSQI) was used to measure the sleep quality before and at 1 month after surgery.

Results: The VAS scores were significantly lower in patients in the combined group, when compared with the other two groups, at 12, 24, 48, and $72 \mathrm{~h}$ after surgery. In addition, the VAS scores at all time points were significantly lower in the FICB group than the control group. The PCIA pressing times were also remarkably lower in the combined group. At 4, 24, 48, and $72 \mathrm{~h}$ after surgery, the serum levels of these inflammatory factors were the lowest in the combined group, and the PSQI scores were significantly lower in the combined group, when compared with the other two groups, while the control group had the highest PSQI scores among the three groups. There was no severe side effects and significant difference observed.
\end{abstract}

Conclusion: FICB combined with DEX reduced the postoperative pain, improved the sleep condition, and decreased the serum levels of inflammatory factors after total hip arthroplasty.

Keywords: Fascia iliaca compartment block, Dexmedetomidine, Pain, Inflammation, Total hip arthroplasty

\footnotetext{
* Correspondence: zhang-y0711@outlook.com

Department of Anesthesiology and Perioperative Medicine, The Second

Hospital of Anhui Medical University, No. 678, Furong Road, Hefei 230000,

China
}

(c) The Author(s). 2020 Open Access This article is distributed under the terms of the Creative Commons Attribution 4.0 International License (http://creativecommons.org/licenses/by/4.0/), which permits unrestricted use, distribution, and reproduction in any medium, provided you give appropriate credit to the original author(s) and the source, provide a link to the Creative Commons license, and indicate if changes were made. The Creative Commons Public Domain Dedication waiver (http://creativecommons.org/publicdomain/zero/1.0/) applies to the data made available in this article, unless otherwise stated. 


\section{Introduction}

Hip fractures are common in elder patients with osteoporosis, which result in a high rate of morbidity, reduced quality of life, and long-term care services [1-4]. Total hip arthroplasty (THA) is a widely adopted surgical method for end-stage hip fractures [5-7]. It has been estimated that approximately 572,000 cases may need THA in 2030 in the USA and that over 6.26 million patients may suffer from hip fractures in 2050 [8-10]. However, despite the wide application of THA for femoral fractures, the postoperative pain management remains as a clinical challenge.

Analgesic methods used in THA usually include the application of anesthetics combined with nerve block methods $[11,12]$. In a meta-analysis, the authors reported that both the femoral nerve block and fascia iliaca block could reduce the pain for patients after total knee and hip arthroplasty [11]. The fascia iliaca compartment block (FICB) is a newly developed method and is mainly used in lower limb surgeries [13]. It has been reported that FICM has superior analgesic effects in surgeries for hip fractures and hip flexion $[14,15]$. It was also found that the FICB could reduce the incidence of delirium in intermediate risk patients [16]. However, most studies have used the FICB as a preoperative treatment, and the application of postoperative FICB remains inadequately reported. Dexmedetomidine (DEX), which is a kind of $\alpha 2$ adrenergic receptor agonist, is mainly used for pain management during or after surgery [17]. It has been reported that the DEX has sedative and analgesic properties and that this could also protect patients from postoperative cognitive dysfunction $[18,19]$.

However, to date, few studies have reported the combined use of DEX and FICB after THA. In the present prospective study, a randomized controlled study was conducted to investigate the efficiency of FICB combined with DEX in postoperative treatment and inflammation management for elder patients after THA. The present study might provide more clinical evidences for the application of the combined use of FICB and DEX in hip surgeries.

\section{Methods and materials}

\section{Patients}

The present randomized controlled open study included a total of 119 elderly patients ( $\geq 60$ years old), who received THA in our hospital from March 2016 to December 2018. All patients who met the inclusion criteria were consecutively enrolled during the study period. The inclusion criteria were as follows: patients who were diagnosed with hip fracture and received THA, patients $\geq 60$ years old, and patients with an American Society of Anesthesiologists (ASA) score of I-III. The diagnosis of hip fracture was confirmed by both X-ray and computed tomography (CT) scan. All surgeries adapted the anterior midline approach. The exclusion criteria were as follows: patients with other fractures; patients with other severe system diseases, such as severe liver, renal, or heart diseases; patients who received analgesics, such as cyclooxygenase inhibitors and opioid receptor agonists, within 1 month before the study; patients with insomnia; and patients with chronic inflammation or pain. All patients provided a signed informed consent. The present study was approved by The Second Hospital of Anhui Medical University.

\section{Anesthesia strategy}

All patients were randomly divided into three groups using a computer-generated list by the SPSS software through a third physician: (1) control group $(n=39)$, patients only received routine general anesthesia; (2) the FICB group $(n=40)$, patients received an additional FICB after surgery; and (3) combined group $(n=40)$, patients received both the pre-treatment of DEX and post-treatment of FICB. For anesthesia induction, these patients were intravenously injected with $1.5 \mathrm{mg} / \mathrm{kg}$ of propofol, $0.03 \mathrm{mg} / \mathrm{kg}$ of midazolam, $0.6 \mathrm{mg} / \mathrm{kg}$ of rocuronium, and $1 \mu \mathrm{g} / \mathrm{kg}$ of remifentanil, followed with endotracheal intubation and mechanical ventilation. Then, $4 \mathrm{mg} / \mathrm{kg}$ of propofol and $0.2-0.5 \mu \mathrm{g} /(\mathrm{kg} \mathrm{min})$ of remifentanil were used for anesthesia maintenance.

For the treatment of DEX, $0.6 \mu \mathrm{g} / \mathrm{kg} / \mathrm{h}$ of DEX was maintained by intravenous injection after anesthesia induction until $30 \mathrm{~min}$ before the end of the surgery, and the other two groups received $0.6 \mu \mathrm{g} / \mathrm{kg} / \mathrm{h}$ of $0.9 \% \mathrm{NaCl}$. For the FICB, the FICB was conducted under ultrasound guidance within 30 min after surgery. Briefly, the iliopsoas, iliac fascia, and fascia lata were observed under ultrasound, and a peripheral nerve plexus stimulation needle was inserted into the iliac fascia to inject $30 \mathrm{ml}$ of $0.2 \%$ ropivacaine. All patients received patient-controlled intravenous analgesia (PCIA) containing $0.12 \%$ bupivacaine + sufentanil citrate of $0.02 \mu \mathrm{g} / \mathrm{kg} / \mathrm{h}$, which were dissolved in $150 \mathrm{ml}$ of normal saline. The single dose of PCIA was limited to $1 \mathrm{ml}$, with a continuous infusion rate $4 \mathrm{ml} / \mathrm{h}$, and the drug locking time was set to $15 \mathrm{~min}$. The routine low molecular weight heparin calcium anticoagulant therapy and antibiotic prevention of infection were performed for all patients.

\section{Measurement of inflammatory factors}

Briefly, peripheral blood samples $(5 \mathrm{ml})$ were collected before the surgery and at $24 \mathrm{~h}$ after surgery. The serum levels of IL-1ß (Cat. no. ab46052, Abcam), IL-6 (Cat. no. ab178013, Abcam), and CRP (Cat. no. LS-F26721, Lifespan Bio.) were determined using commercial enzymelinked immunosorbent assay (ELISA) kits. 


\section{Data collection}

The patient characteristics, including age, gender, body mass index (BMI), and fracture type, were recorded. The resting and moving visual analog scale (VAS) scores were all measured at $12,24,48$, and $72 \mathrm{~h}$ after surgery. The PCIA pressing time within $48 \mathrm{~h}$ after surgery was also recorded. The Pittsburgh sleep quality index (PSQI) was used to measure the sleep quality before and at 1 month after surgery [20]. The side effects within 3 days after surgery were also recorded.

\section{Statistical analysis}

The measurement data was expressed as mean \pm standard deviation (SD) for continuous data. Counting materials were compared using chi-square test. The comparison among three or more groups were conducted using one-way analysis of variance (ANOVA), followed by Tukey post hoc test. $P<0.05$ was considered statistically significant. All calculations were performed using SPSS 20.0.

\section{Results}

\section{Characteristics of all patients}

During the study period, four patients quit the study, while three patients were excluded due to bad compliance. Finally, 112 patients were maintained, with 37 patients in the control group, 37 patients in the FICB group, and 38 patients in the combined group (Fig. 1).
Among all patients, 47 patients had intertrochanteric fracture of the femur, while 65 patients had fracture of the femoral neck. However, no significant difference was found for age, gender, BMI, and fracture type among the different groups of patients (Table 1).

\section{The combined use of DEX and FICB in pain condition after THA}

The pain condition for the different groups of patients was determined by the VAS score and PCIA pressing times. As shown in Fig. 2, both the resting and moving VAS scores were significantly lower in patients of the combined group when compared with the other two groups at $12,24,48$, and $72 \mathrm{~h}$ after surgery $(P=0.000$, for all comparisons within the three groups at all points). Furthermore, the VAS scores at all time points were significantly lower in the FICB group when compared with the control group $(P=0.000$, for all comparisons within the three groups at all points). Similar results were also observed for PCIA pressing times within $48 \mathrm{~h}$ after surgery.

\section{Effect of the combined use of DEX and FICB on inflammatory factors after THA}

The inflammatory factors before and at $24 \mathrm{~h}$ after surgery were determined. The results revealed that the serum levels for all factors were significantly upregulated at $24 \mathrm{~h}$ after surgery for all groups when compared with

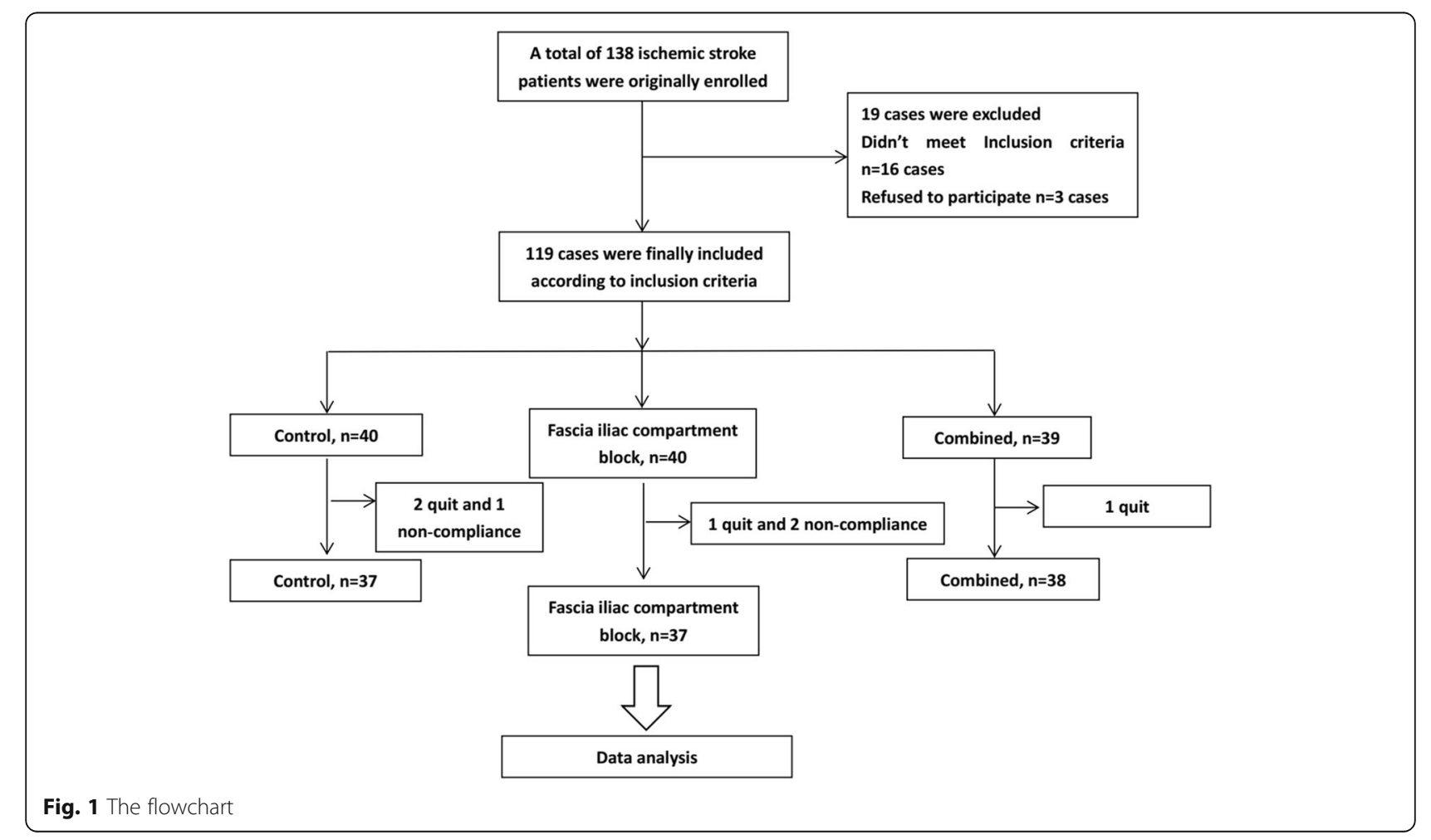


Table 1 Patients characteristics

\begin{tabular}{|c|c|c|c|c|}
\hline Variables & Control, $n=37$ & $\mathrm{FICB}, n=37$ & Combined, $n=38$ & $P$ value \\
\hline Age, year & $70.00 \pm 5.69$ (62 79) & $70.05 \pm 5.52(60 \sim 78)$ & $67.37 \pm 6.21(60 \sim 79)$ & 0.077 \\
\hline Gender, female (\%) & $12(32.43)$ & $11(29.73)$ & $13(34.21)$ & 0.792 \\
\hline BMI $\left(\mathrm{kg} / \mathrm{m}^{2}\right)$ & $23.05 \pm 2.89$ & $23.24 \pm 3.13$ & $22.81 \pm 3.02$ & 0.828 \\
\hline Fracture type, $n(\%)$ & & & & 0.926 \\
\hline Fracture of femoral neck & $21(56.76)$ & $22(59.46)$ & $22(57.89)$ & \\
\hline Intertrochanteric fracture of femur & $16(43.28)$ & $15(40.54)$ & $16(42.11)$ & \\
\hline
\end{tabular}

$B M I$ body mass index

the levels before surgery. Meanwhile, at $24 \mathrm{~h}$ after surgery, the serum levels of these inflammatory factors were the lowest in the combined group, when compared with the other two groups, and the difference was statistically significant $(P=0.000$, for all comparisons; Table 2). Patients in the control group exhibited remarkably higher serum levels of inflammatory factors, when compared with the combined group and FICB group $(P=0.000$, for all comparisons).

\section{Effect of the combined use of DEX and FICB on sleep quality index and the side effects}

Lastly, the combination of DEX and FICB on sleep quality index and the side effects were measured. As shown in Table 3 , the PSQI scores were significantly lower in the combined group than in the other two groups, while patients in the control group had the highest PSQI scores among the three groups $(P=$ 0.000 ). The side effects in all groups were also recorded, and no severe side effects or significant difference was observed.

\section{Discussion}

Pain management after THA is a main problem that affects a patient's postoperative recovery. At present, several strategies have been reported to enhance the postoperative recovery and reduce the pain, such as oral and intramuscular analgesics, local block at the operative site, nerve block, intravenous controlled analgesia, and epidural controlled analgesia [21, 22]. Among these

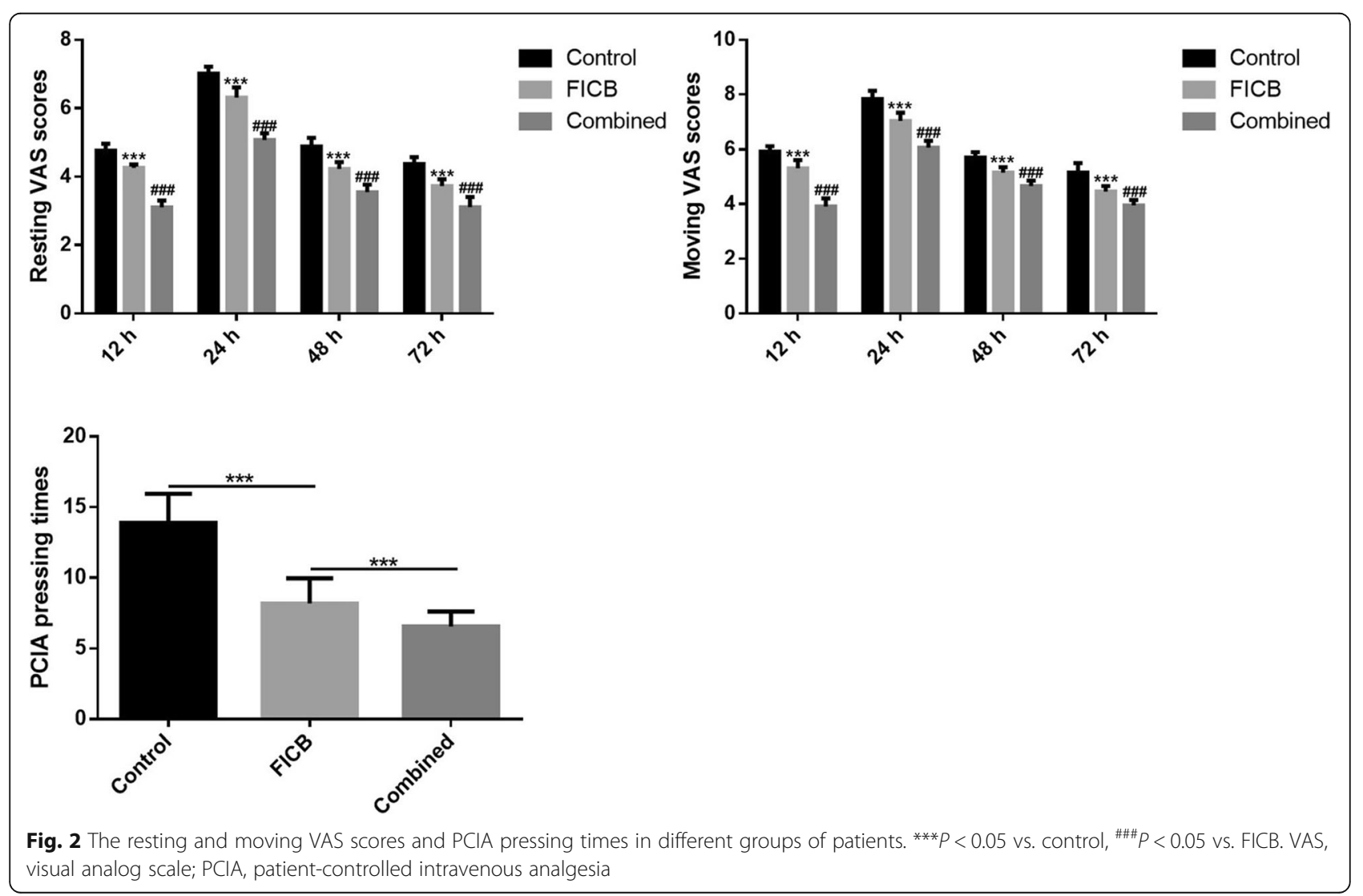


Table 2 Effect of combination use of DEX and fascia iliaca compartment block on inflammatory factors after total hip arthroplasty

\begin{tabular}{lllll}
\hline Variables & & Control, $n=37$ & FICB, $n=37$ & Combined, $n=38$ \\
\hline IL-6, pg/ml & Before & $84.89 \pm 2.88$ & $84.26 \pm 3.39$ & $85.64 \pm 2.90$ \\
& $24 \mathrm{~h}$ & $173.45 \pm 12.04$ & $163.48 \pm 6.90^{\mathrm{a}}$ & $137.62 \pm 8.18^{\mathrm{a}, \mathrm{b}}$ \\
$\mathrm{IL}-1 \beta, \mathrm{pg} / \mathrm{ml}$ & Before & $34.34 \pm 2.94$ & $34.33 \pm 3.18$ & $34.99 \pm 2.91$ \\
& $24 \mathrm{~h}$ & $54.15 \pm 3.05$ & $50.22 \pm 3.32^{\mathrm{a}}$ & $45.50 \pm 2.87^{\mathrm{a}, \mathrm{b}}$ \\
$\mathrm{CRP}, \mathrm{mg} / \mathrm{L}$ & Before & $7.51 \pm 0.92$ & $7.63 \pm 0.83$ & $7.52 \pm 0.74$ \\
& $24 \mathrm{~h}$ & $41.53 \pm 1.38$ & $36.45 \pm 2.26^{\mathrm{a}}$ & $34.41 \pm 2.59^{\mathrm{a}, \mathrm{b}}$ \\
\hline
\end{tabular}

IL interleukin, CRP C-reactive protein

${ }^{\mathrm{a}} P<0.05$ vs. control

${ }^{\mathrm{b}} \mathrm{P}<0.05$ vs. FICB

methods, the FICB is a newly developed method, in which local anesthetics are injected into the FICB and is mainly used in surgeries for the lower limbs [16]. Although several studies have reported the application of FICB for lower limb surgeries, few studies have focused on the combined use of the FICB and DEX in THA. In the present study, it was demonstrated that the combination of FICB and DEX could reduce postoperative pain, improve sleep condition, and decrease serum inflammatory factors after THA.

The FICB has been used in several types of surgeries. Yu et al. compared a continuous femoral nerve block and a continuous fascia iliaca compartment block in THA surgery for elder patients and found that the continuous fascia iliaca compartment block has a better effect on pain condition [23]. Williams et al. revealed that standard analgesia combined with FICB significantly reduced the VAS scores of patients with femoral neck fractures after surgery [24]. In a meta-analysis, Steenberg et al. reported that the FICB was better than opioids during movement and has lower preoperative analgesia consumption, a longer time for first request, and a reduced time to perform the spinal anesthesia [13]. In the present study, it was also found that the FICB treatment reduced postoperative pain, improved the sleep condition, and decreased the serum inflammatory factors. Inflammatory factors, such as IL-1 $\beta$, IL-6, and CRP, have also been reported to be elevated in surgeries, including THA. In an earlier study, it was demonstrated that the levels of IL-6 and TNF- $\alpha$ increased after THA [25]. It was also reported that CRP levels remained high during the entire observational period after surgery for THA [26]. In addition, studies have found that DEX could also reduce the levels of IL-1 $\beta$, IL-6, and CRP, both in vivo and in vitro [27-29]. All these results were consistent with the present findings.

DEX is presently widely used for pain postoperative management in various surgeries, including THA. It has been reported that preoperative intravenous DEX can prevent tourniquet-induced hypertension in orthopedic operations [30]. In a case report, it was reported that DEX was effective as a sedative and analgesic for a total hip replacement patient [31]. In a recent research, Mei et al. reported that the intraoperative sedation of DEX was better than propofol in THA for elder patients [32]. In addition, the combined use of DEX and other block anesthetic methods have also been reported in several studies. It was reported that DEX could be used as an adjuvant to $2 \%$ lignocaine in an infraorbital nerve block [33]. Another study also revealed that DEX could reduce the morphine consumption, VAS scores, and incidence of postoperative nausea/vomiting [34]. In the present study, it was found that DEX could enhance the pain measurement effects of the FICB. Furthermore, this also reduced the serum levels of inflammatory factors and improved the sleep condition, with no obvious side effects. However, the present study has some limitations. First, the study sample size was limited. Second, all patients were from a single center. Third, the long-term effects of the method were not investigated. Hence, further studies are needed to confirm these results.

\section{Conclusion}

In conclusion, a prospective randomized control study was conducted to investigate the efficiency of FICB combined with DEX after THA. It was observed that the

Table 3 Effect of combination use of DEX and fascia iliaca compartment block on sleep quality index and the side effects

\begin{tabular}{lllll}
\hline Variables & Control, $n=37$ & FICB, $n=37$ & Combined, $n=38$ & $12.00 \pm 1.34$ \\
\hline PSQI before & $12.08 \pm 1.64$ & $11.86 \pm 1.29$ & $6.00 \pm 1.47$ & 0.806 \\
PSQI after & $10.24 \pm 1.23$ & $7.76 \pm 1.48$ & & 0.000 \\
Side effects, $n$ (\%) & & & $1(2.6)$ & 0.731 \\
$\quad$ Nausea & $2(5.4)$ & $1(2.7)$ & $2(5.2)$ \\
$\quad 1(2.7)$ & $1(2.7)$ & $1(2.6)$ \\
$\quad$ Emesis & $1(2.7)$ & $1(2.7)$ & \\
\hline
\end{tabular}


FICB combined with DEX reduced the postoperative pain, improved the sleep condition, and decreased the serum levels of inflammatory factors after THA. The present study might provide more clinical evidences for the application of the FICB and DEX in the postoperative treatment of THA.

\author{
Abbreviations \\ CRP: C-reactive protein; DEX: Dexmedetomidine; ELISA: Enzyme-linked \\ immunosorbent assay; FICB: Fascia iliaca compartment block; PCIA: Patient- \\ controlled intravenous analgesia; PSQI: Pittsburgh sleep quality index; \\ THA: Total hip arthroplasty; VAS: Visual analog scale
}

\section{Acknowledgements}

This work is supported by The Second Hospital of Anhui Medical University.

\section{Authors' contributions}

Each author has made an important scientific contribution to the study and has assisted with the drafting or revising of the manuscript. All authors have read and approved the final manuscript.

\section{Ethics approval and consent to participate}

Ethical approval was given by The Second Hospital of Anhui Medical University. All patients signed the written informed consent.

\section{Consent for publication}

All of the authors have consent to publish this research.

\section{Competing interests}

The authors declare that they have no competing interests.

Received: 9 September 2019 Accepted: 15 January 2020

Published online: 10 February 2020

\section{References}

1. Parker M, Johansen A. Hip fracture. BMJ. 2006;333(7557):27-30.

2. Roberts KC, Brox WT, Jevsevar DS, Sevarino K. Management of hip fractures in the elderly. J Am Acad Orthop Surg. 2015;23(2):131-7.

3. Jantzen C, Madsen CM, Lauritzen JB, Jørgensen HL. Temporal trends in hip fracture incidence, mortality, and morbidity in Denmark from 1999 to 2012. Acta Orthop. 2018;89(1):1.

4. Amin NH, Hutchinson HL, Sanzone AG. Management of acute hip fracture N Engl J Med. 2018:378(10):971

5. Homma Y, Baba T, Sano K, Ochi H, Matsumoto M, Kobayashi H, et al. Lateral femoral cutaneous nerve injury with the direct anterior approach for total hip arthroplasty. Int Orthop. 2016;40(8):1587-93.

6. Meermans G, Konan S, Das R, Volpin A, Haddad FS. The direct anterior approach in total hip arthroplasty. Bone Joint J. 2017;99-B(6):732-40.

7. Berend KR, Lombardi JA, Berend ME, Adams JB, Morris MJ. The outpatient total hip arthroplasty : a paradigm change. Bone Joint J. 2018;100-B(1 Supple A):31.

8. Sun X, Dong Q, Zhang YG. Intravenous versus topical tranexamic acid in primary total hip replacement: a systemic review and meta-analysis. Medicine. 2016:95(50):10-8

9. Liao K-M, Lu H-Y. A national analysis of complications following total hip replacement in patients with chronic obstructive pulmonary disease. Medicine. 2016;95(12):e3182.

10. Cooper C, Campion G, Melton LJR. Hip fracture in the elderly: a worldwide projection. Osteoporos Int. 1992;2(6):285-9.

11. Wang $X$, Sun $Y$, Wang $L$, Hao $X$. Femoral nerve block versus fascia iliaca block for pain control in total knee and hip arthroplasty: a meta-analysis from randomized controlled trials. Medicine. 2017;96:27.

12. Jørgensen CC, Petersen M, Kehlet H, Aasvang EK. Analgesic consumption trajectories in 8.975 patients 1-year after fast-track total hip or knee arthroplasty. Eur J Pain. 2018:22(8):1428-38.

13. Steenberg J, Møller AM. Systematic review_effects of fascia iliaca compartment block on hip fractures before operation. Br J Anaesth. 2018; 120(6):1368-80.

14. Newman B, Mccarthy L, Thomas PW, May P, Horn K. A comparison of preoperative nerve stimulator-guided femoral nerve block and fascia iliaca compartment block in patients with a femoral neck fracture. Anaesthesia. 2013:68(9):899-903.

15. Klukowski M, Kowalczyk R, Górniewski G, Łęgosz P, Trzebicki J. Iliac fascia compartment block and analgesic consumption in patients operated on for hip fracture. Ortop Traumatol Rehabil. 2017;19(5):451-9.

16. Monzon DG, Iserson KV, Vazquez JA. Single fascia iliaca compartment block for post-hip fracture pain relief. J Emerg Med. 2007;32(3):257-62.

17. Bajwa S, Kulshrestha A. Dexmedetomidine: an adjuvant making large inroads into clinical practice. Ann Med Health Sci Res. 2013;3(4):475-83.

18. Keating GM. Dexmedetomidine: a review of its use for sedation in the intensive care setting. Drugs. 2015:75(10):1119-30.

19. Deiner S, Luo X, Lin H-M, Sessler DI, Saager L, Sieber FE, et al. Intraoperative infusion of dexmedetomidine for prevention of postoperative delirium and cognitive dysfunction in elderly patients undergoing major elective noncardiac surgery. JAMA Surg. 2017;152(8):e171505.

20. Chen AF, Orozco FR, Austin LS, Post ZD, Deirmengian CA, Ong AC. Prospective evaluation of sleep disturbances after total knee arthroplasty. J Arthroplast. 2016;31(1):330-2.

21. Kehlet H, Dahl JB. Anaesthesia, surgery, and challenges in postoperative recovery. Lancet. 2003;362(9399):1921-8.

22. Allvin $\mathrm{R}$, Berg $\mathrm{K}$, Idvall $\mathrm{E}$, Nilsson U. Postoperative recovery: a concept analysis. J Adv Nurs. 2010:57(5):552-8.

23. Yu B, He M, Cai GY, Zou TX, Zhang N. Ultrasound-guided continuous femoral nerve block vs continuous fascia iliaca compartment block for hip replacement in the elderly: A randomized controlled clinical trial (CONSORT). Medicine (Baltimore), 2016: 95(42):e5056.

24. Williams H, Paringe V, Shenoy S, Michaels P, Ramesh B. Standard preoperative analgesia with or without fascia iliaca compartment block for femoral neck fractures. J Orthop Surg. 2016;24(1):31-5.

25. Høgevold HE, Lyberg T, Kähler H, Haug E, Reikerås $\mathrm{O}$. Changes in plasma IL$1 \beta$, TNF- $\alpha$ and IL-6 after total hip replacement surgery in general or regional anaesthesia. Cytokine. 2000;12(7):1156-9.

26. Bouaicha S, Blatter S, Moor BK, Spanaus K, Dora C, Werner CM. Early serum procalcitonin level after primary total hip replacement. Mediat Inflamm. 2013:2013(23-24):927636 (2013-5-13)

27. Tanabe K, Matsushima-Nishiwaki R, Kozawa O, lida H. Dexmedetomidine suppresses interleukin-1 $\beta$-induced interleukin-6 synthesis in rat glial cells. Int J Mol Med. 2014;34(4):1032-8.

28. Kang SH, Kim YS, Hong TH, Chae MS, Cho ML, Her YM, et al. Effects of dexmedetomidine on inflammatory responses in patients undergoing laparoscopic cholecystectomy. Acta Anaesthesiol Scand. 2013;57(4):480-7.

29. Li Y, He R, Chen S, Qu Y. Effect of dexmedetomidine on early postoperative cognitive dysfunction and peri-operative inflammation in elderly patients undergoing laparoscopic cholecystectomy. Exp Ther Med. 2015;10(5):1635-42.

30. Lu Y, Zhang Y, Dong CS, Yu JM, Wong GT. Preoperative dexmedetomidine prevents tourniquet-induced hypertension in orthopedic operation during general anesthesia. Kaohsiung J Med Sci. 2013;29(5):271-4.

31. Dhansura T, Kapadia S, Gandhi S. Dexmedetomidine as sedative and analgesic in a patient of sickle cell disease for total hip replacement. Indian J Anaesth. 2013:57(4):425-6.

32. Mei B, Meng G, Xu G, Cheng X, Chen S, Zhang Y, Zhang M, Liu X, Gu E. Intraoperative Sedation With Dexmedetomidine is Superior to Propofol for Elderly Patients Undergoing Hip Arthroplasty: A Prospective Randomized Controlled Study. Clin J Pain. 2018;34(9):811-17.

33. Khandaitkar S, Kolte V, Shenoi SR, Budhraja N. A clinical study to determine the efficacy of 7 ppm dexmedetomidine as an adjuvant to $2 \%$ lignocaine in infraorbital nerve block. Br J Oral Maxillofac Surg. 2016:54(9):997-1000.

34. Mohta M, Kalra B, Sethi AK, Kaur N. Efficacy of dexmedetomidine as an adjuvant in paravertebral block in breast cancer surgery. J Anesth. 2016; 30(2):252-60.

\section{Publisher's Note}

Springer Nature remains neutral with regard to jurisdictional claims in published maps and institutional affiliations. 